\title{
A Mobile Cancer Follow-up Care Plan for Patient Empowerment
}

Kerstin Denecke ${ }^{1 *}$, Tim L Dorner ${ }^{1}$, Rea Iseli ${ }^{1}$, Sarah Mele ${ }^{1}$, Mauro Tschanz ${ }^{1}$, Carsten T Viehl' ${ }^{2}$, Michael Lehmann ${ }^{1}$ and Thomas Bürkle ${ }^{1}$

${ }^{1}$ Berner Fachhochschule, Biel, Switzerland

${ }^{2}$ Spitalzentrum Biel, Biel, Switzerland

\begin{abstract}
An increasing prevalence of cancer requires new ways of supporting outpatients and improving their compliance to treatment. Initial mostly surgical treatment is followed by long-term surveillance to detect and treat cancer recurrence timely. Depending on the tumor stage, the frequencies of follow-up appointments differ and the patient has to remember to make these appointments at the right time. Currently, the patient receives a paper-based schema indicating the follow-up schedule. Since this document is difficult to understand or may get lost, patients are missing the follow-up appointments. This paper illustrates our attempt at exploiting mHealth to support the empowerment and awareness in outpatients by a mobile, bilingual app that supports variable cancer follow-up schedules. Installed on a mobile device, the app reminds the patient to schedule his appointments timely and provides additional information about disease and treatment. Equipped with additional functionalities for monitoring vital parameters, the app can be used for continuous monitoring. The usability was studied with nine persons. We conclude that the app provides a good basis for a successful product after realizing some extensions and improvements of the usability.
\end{abstract}

Keywords: After-treatment; Rectal tumor; Supportive cancer care; Patient empowerment; Mobile application

\section{Introduction}

An increasing prevalence is scored by cancer along with a corresponding rise in the survivor's number, so that cancer itself is considered a chronic condition [1]. Chronic diseases result in a large management burden in outpatients who need to comply with their follow-up examinations and treatments over an extended time period. In tumor patients, the long-time surveillance following the hospitalization is very important for early detection of tumor recurrence or metastases. Guidelines for cancer follow-up exist for many cancer types in the different countries. Here, we focus on colorectal cancer which caused 11,344 hospital treatments in 2014 in Switzerland [2]. The Swiss societies for gastroenterology and visceral surgery published consensus recommendations for aftercare after curatively operated colorectal carcinoma [3]. Studies showed that the quality of surveillance after curative surgery for colon cancer among a cohort of Swiss patients is inadequate [4]. One reason is that it is still challenging for patients to remember their appointments and there is no other reminder system in place that could help. Viehl state that "further education of health care professionals and patients regarding the potential life-saving benefits of surveillance is imperative" [4]. We believe that mHealth has the potential of improving this situation.

During the past decades people became more technology oriented thanks to a large scale distribution of mobile devices. The introduction of mobile applications in healthcare led to the definition of mHealth which is the delivery of health related services through the use of mobile devices. Mobile apps have been proven to be useful for health professionals and patients [5] as they have potentials to increase access to evidence-based care, better inform consumers of care and enhance care after formal treatment [6]. Today, several apps for cancer care are available: Nachsorge-App [7] and My Care Plan [8] are designed for health care teams to coordinate the followup care and monitor any ongoing symptoms. The "Krebshilfe" app [9] offers extensive information on topics such as side effects, diet, rehabilitation, cancer and occupation or the emergency aid of the Austrian Cancer Aid. The mobile application HeNeA supports patient education, self-monitoring, collaboration with the specialist and social interaction with peers [10].
In contrast to these existing apps, we aim for a system that supports personalized cancer follow-up schemes and provides reminders for making the required appointments. To the best of our knowledge, there is no app available that is using standardized follow-up schemes. Thus, our goals are as follows:

- The app should remind the patient to the necessary appointments over the next 5 years based on the individual TNM (tumornodes-metastases) classification. The selection of the correct surveillance schema is thus realized semi-automatically.

- The patient should be better informed about his progress. Therefore, the patient should be enabled to record and understand the results of his follow-up checks in the app.

\section{Methods}

A requirements analysis was performed using unstructured interviews with three consultant surgeons of the Spitalzentrum Biel, the local hospital in Biel/Switzerland. Initially, the chief physician was introducing the challenges in cancer treatment. Then, the three physicians were asked independently from each other on their ideas and requirements for a supporting tool. Afterwards, the requirements were consolidated.

A usability test with the prototype of the app was carried out by means of a survey consisting of 10 questions where the subjects had to select an item on a scale of 1 to 5 (from "does not apply" to "applies totally"). The test persons were healthy persons recruited from the authors' social environment, i.e. not diagnosed with rectal cancer. A total of nine people tested the app; five of them are male and four

*Corresponding author: Kerstin Denecke, Berner Fachhochschule, Quellgasse 21, 2501 Biel, Switzerland, Tel: +41 3232167 94; E-mail: kerstin.denecke@bfh.ch

Received May 31, 2017; Accepted June 21, 2017; Published June 26, 2017

Citation: Denecke K, Dorner TL, Iseli R, Mele S, Tschanz M, et al. (2017) A Mobile Cancer Follow-up Care Plan for Patient Empowerment. J Health Med Informat 8 : 268. doi: 10.4172/2157-7420.1000268

Copyright: () 2017 Denecke K, et al. This is an open-access article distributed under the terms of the Creative Commons Attribution License, which permits unrestricted use, distribution, and reproduction in any medium, provided the original author and source are credited. 
female. Eight of them use their smartphone daily. One person uses the smartphone only about once a week. The age distribution was as follows:

- 2 people between 30 and 40 years.

- 6 people between 50 and 60 years.

- 1 person between 70 and 80 years.

The authors of this article were not involved in the usability evaluation for reasons of independence. The basis for the usability evaluation was defined by a specially created set of usability criteria considering interaction processes, interface design, and comprehensibility of content. The app was tested on devices that have been provided to them instead of installing the app on their personal mobile phones. The user was confronted with a test scenario comprising eight tasks (e.g. entering vital parameter, export of the timeline as pdf). Using test scenarios is a well-accepted mean in usability studies [11].

The number of clicks was measured and the time needed to fulfil a task. In addition to the tasks, we asked on feedback whether the app and functionalities are understandable etc. by the above mentioned questionnaire. Beyond, the app was shown and discussed with a patient suffering from rectal cancer and a physician and their feedback was collected. For the usability study, no ethics approval was required since no personal health data was collected by the app.

\section{System Architecture and Functionalities of the App}

In this section, we first explain the follow-up schema that forms the basis for the timeline shown in the application. Then, we describe the collected requirements and the implemented functionalities. Afterwards, the system architecture for realizing these requirements and functionalities is introduced.

\section{Follow-up schema}

The basis for our work was the post-surgery follow-up schema of the Swiss Society for Gastroenterology [3]. This follow-up schema is currently given to the patient in paper form. The document contains several schemata to be applied depending on the tumor stage (TNMvalues). The TNM classification of the carcinoma of the patient and the selection of the carcinoma type determine the pattern.

We included three schemata for the app development for colon carcinoma (stage 1: T1/T2 N0, stage 2 and 3: T3/4 or N+, M0) and rectum carcinoma (all stages: T1-4, N+/-, M0, shown in Table 1). More advanced tumor stages have been excluded in the first prototype because they need an individualized follow-up schema.

\section{Requirements}

The collected requirements concern the selection of the schema, the reminder function and additional features. They are listed in Table 2 together with information on the status of implementation in the prototype.

\begin{tabular}{|l|c|c|c|c|c|c|c|}
\hline Rectum carcinoma & & \multicolumn{7}{|c|}{ Months post operation } & \\
\hline T1-4, N+/-,M0 & 6 & 12 & 18 & 24 & 36 & 48 & 60 \\
\hline Examination, CEA-Titer & $\begin{array}{l}\text { Every 3 } \\
\text { months }\end{array}$ & $\begin{array}{l}\text { Every } 6 \\
\text { months }\end{array}$ & & + & + \\
\hline Colonoscopy & & + & & & & + & \\
\hline Flexible endoscopy & + & & + & + & & & \\
\hline Endosonography or MRI Pelvis & + & + & + & + & & & \\
\hline CT Thorax Abdomen Pelvis & & + & & + & + & + & + \\
\hline
\end{tabular}

Table 1: Example of follow-up schedule (for rectal cancer, all stages).

\section{Functionalities}

The app provides the following features: The patient is enabled to enter the relevant data on tumor (TNM-values) and carcinoma as provided by the physician at discharge on paper (Figure 1). This is requested only when the app is started the first time. The appointments are generated automatically based on the entered TNM-values and displayed in a timeline (Figure 2). At the beginning, the dates are only marked with the time period, i.e. the month in the respective year. Once an appointment has been made, the patient can enter and adjust the appointment. Contacts (e.g. GP, specialist) can be stored and called directly from the application. Intentionally, we did not use the contacts of the smartphone for data privacy and security reasons. The app integrates information from official cancer care sources. Currently, the system links to the Krebshilfe.ch website (cancer care organization). Vital parameters can be entered manually by the patient for continuous monitoring of the heart rate and body weight. These parameters are displayed graphically to show trends or significant changes. An export function allows sending the data via E-mail.

\section{System architecture}

The system consists of three components shown as system architecture in Figures 3 and 4 . The Client provides the different views or user interfaces for login, data entry, information access etc.

\begin{tabular}{|c|c|}
\hline Requirement & Implemented in the prototype \\
\hline \multicolumn{2}{|c|}{ Enter relevant data for selecting the schema } \\
\hline $\begin{array}{l}\text { enter type of carcinoma (rectal or colon } \\
\text { carcinoma) }\end{array}$ & yes \\
\hline enter data of surgery & yes \\
\hline enter completeness of colonoscopy & yes \\
\hline enter TNM stage & yes \\
\hline \multicolumn{2}{|l|}{ Schedule and reminder } \\
\hline $\begin{array}{l}\text { show all dates according to the schema in } \\
\text { a timeline }\end{array}$ & yes \\
\hline user can select dates can completed & yes \\
\hline reminder function & yes \\
\hline \multicolumn{2}{|l|}{ Additional features } \\
\hline contact family doctor by E-mail & $\begin{array}{l}\text { changed to contact by telephone } \\
\text { upon request }\end{array}$ \\
\hline $\begin{array}{l}\text { vital parameter such as pulse and weight } \\
\text { can be entered in the best case uploaded } \\
\text { from external gadgets }\end{array}$ & $\begin{array}{l}\text { data can be entered, but not } \\
\text { uploaded automatically }\end{array}$ \\
\hline show relevant health information & yes \\
\hline encrypted data storage & yes \\
\hline
\end{tabular}

Table 2: Collected requirements and their status in the prototype.

\section{SINA - Follow-up Care App}

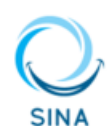

Patient:

Lena Gerber

TNM-Staging:

\begin{tabular}{l|l|l}
$\mathrm{T}: 1$ & $\mathrm{~N}: 1$ & $\mathrm{M}: 0$
\end{tabular}

Carcinoma:

Colon Carcinoma

Date of surgery:

15 December 2016

Colonoscopy complete? Yes $\square \quad$ NolX

Figure 1: Form that is given to patient at discharge. It contains all relevant data to be entered into the app for initialization. 
Citation: Denecke K, Dorner TL, Iseli R, Mele S, Tschanz M, et al. (2017) A Mobile Cancer Follow-up Care Plan for Patient Empowerment. J Health Med Informat 8: 268. doi: 10.4172/2157-7420.1000268

The app is running on Apple iOS and Android 7.0 and was designed to support German and French language. It was developed using the Ionic framework for hybrid apps (https://ionicframework.com/) with AngularJS and Apache Cordova. The Ionic framework supports connecting to the MIDATA IT Platform (https://www.midata.coop). The client connects with the MIDATA web services for authentication and connection to the MIDATA Database. The MIDATA database contains the patient data, i.e. the data collected by Figure 5; the app is transmitted to the MIDATA database to avoid local data storage on the mobile system of the user. The user needs a MIDATA account to enable the app to access the data on MIDATA. When changing the mobile phone, the stored data can be directly imported from the user account.

The data exchange between the app and the platform MIDATA is realised using a web service provided by MIDATA. Due the multiply

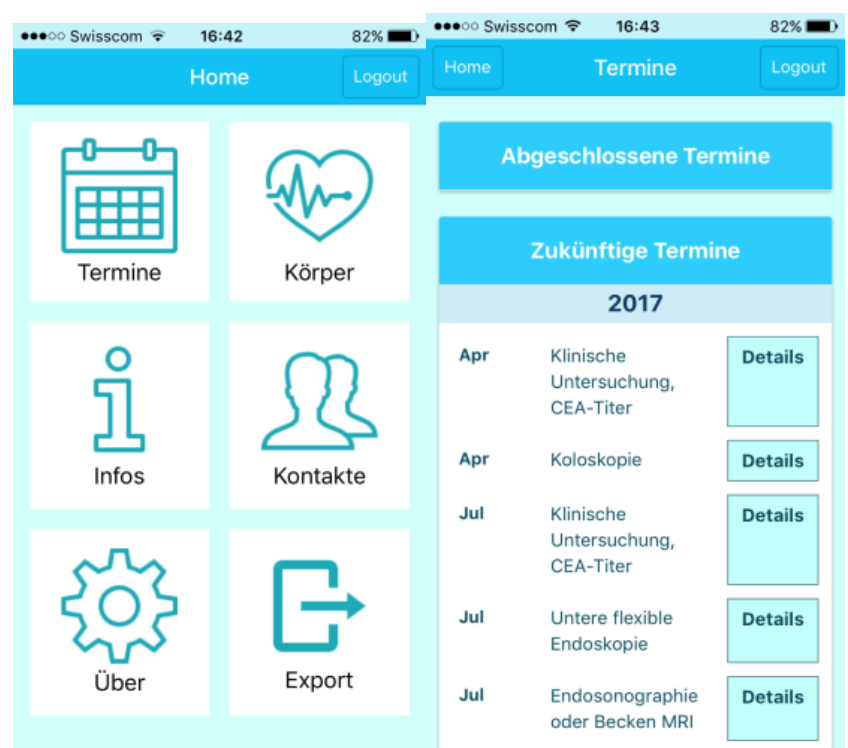

Figure 2: The app: Home screen (left) with appointments (Termine), vital parameters (Körper), information, contacts (Kontakte), About (Über) and export functionality. Right: Appointment view, split into completed appointments (Abgeschlossene Termine) and future appointments (Zukünftige Termine).

\begin{tabular}{|lll}
\hline Home Körper Lout \\
\hline
\end{tabular}
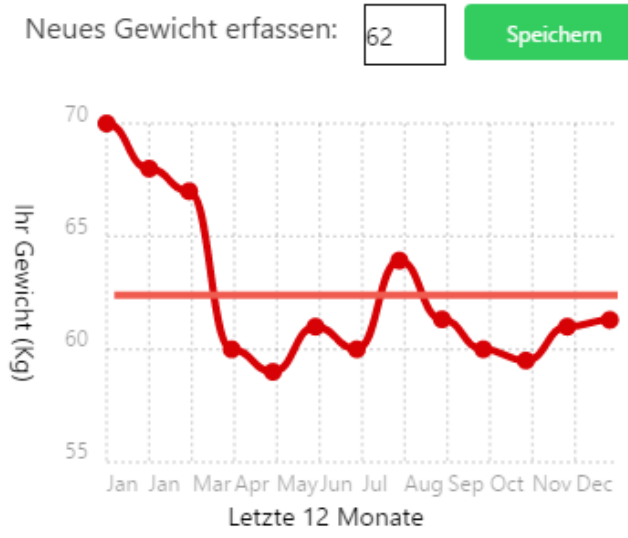

Figure 3: Vital parameter of the patient

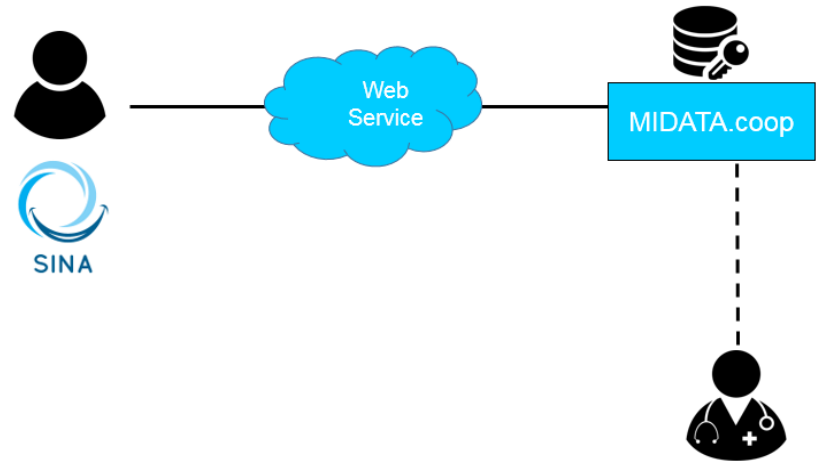

Figure 4: System architecture: The app consists of a client as interaction interface with the user. Through a web service, authentication and data transfer with MIDATA is enabled. Data can be accessed from MIDATA by authorized physicians.

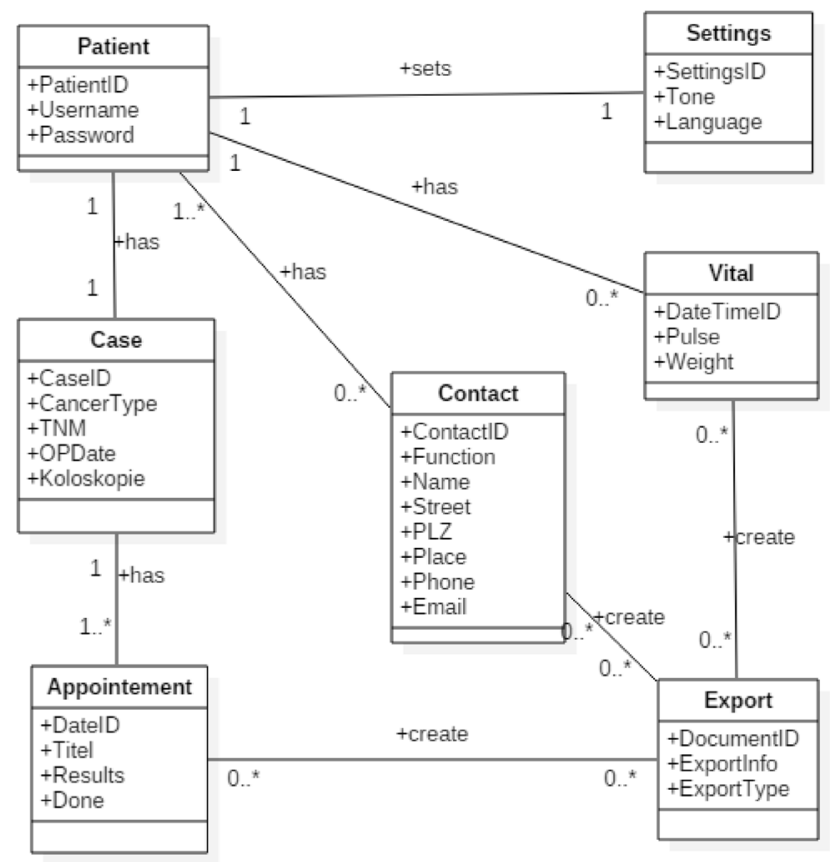

Figure 5: Shows the database structure. A patient object has specific settings several vital parameters, contacts and one case. The case object encapsulates the relevant information for selecting the follow-up schema.

encrypted data, MIDATA ensures a high safety standard, which is indispensable for personal medical data. The encryption of the key guarantees that the data is exclusively controlled by his owner. Through the loss of the key the data can no longer be decrypted and are thus lost. The data collected by the app and transferred to the MIDATA server can be made available by the patient for anonymized use in studies or for monitoring purposes of the personal health care team.

\section{Results}

\section{Usability test results}

The results of the usability test demonstrated that both, test persons that use their smartphone daily or those who use it "only" weekly are able to use the app without major problems. All test persons could successfully complete all tasks within 15-30 min. The users had 
sometimes difficulties to select the tumor stage and operation dates in the app. Picking the right value proved to be tedious and took an excessive amount of time. Also, the font size turned out to be too small: The test persons had to zoom in to read. The schedule listing the appointments was considered confusing and counter-intuitive by at least three people. The information about data protection was part of a so-called "Hamburger button" (typically used in Android), which was not intuitive for users of an iPhone. The vital parameters could be entered without problems. The save icon turned out to be not intuitive and was replaced afterwards. Table 3 shows the usability questionnaire and the number of replies per item and scale value.

\section{User feedback}

The physician was overall impressed by the prototype and confirmed the usefulness of the implemented functionalities. He suggested some extensions, such as a free text field for specifying the carcinoma (left, right) and a warning that the app cannot be applied for tumor follow-up when metastases were found (M1 in TNM). Further, he mentioned several ideas for representing the data: the diagram of the weight should have an initial value. Some texts and wordings should be improved and adapted to the patient language. Entering additional data on the disease should be enabled.

Feedback from the patient concerned a potential user guide. The timeline generation and the appointment list require some explanation. This could be realized by pop ups with corresponding information. Beyond, it turned out that the patient is interested in storing the examination results (e.g. blood, CEA-Titer) for each appointment. Collection of vital data should be preferably realized by integrating data collected from wearables or smart devices.

\section{Discussion}

In this paper, we presented an app to support cancer patients in the follow-up care process by providing a guideline based care plan, information and additional features for monitoring health. The benefits and limitations of the system and the overall approach are discussed in the following. Further, limitations of the study design are presented.

\section{Benefits and limitations of the approach}

The Institute of Medicine provided in 2006 recommendations for improving follow-up care for cancer patients. They claimed that all patients should be equipped with a comprehensive treatment summary and follow-up care plan [12]. Further, Groen et al. [13] found out that among others educational services including care plan services brought to the patient through IT services may contribute to patient empowerment. They conclude that it is important to consider the needs of a patient when developing IT services, and to create services that are attractive and easy to use. With the concept and prototype presented in this work, we contribute in addressing these needs. Information on the patient's health can be shared easily with the care team through the data availability at MIDATA. This can improve communication among care providers and enables a continuous monitoring. The reminder function ensures that the patient is aware of follow-up examinations. The system can provide an additional added value when the patient can enter or upload examination results. This could again improve the monitoring process [14].

Geng and Sahit state that cancer survivors do not often receive proper follow-up care despite existing guidelines, but that patient empowerment is crucial for surviving and post-care [15]. They found out that only few apps are designed for cancer survivors. The seven apps they identified support the following functionalities: 1) Providing basic information and patient education information sources, 2) Creating a care plan, self-enter patient medical history and manage medical documents; 3) Tracking health data and lifestyle (diet, weight, and exercise routine); 4) Sending appointment and medication reminders by text messaging; 5) Sending text reminders for daily care, health and symptom assessment; and 6) Offering social media to potentially support better information exchange among survivors.

Pandey et al. found out that there is a lack of cancer-related applications with scientifically backed data $[16,17]$. In contrast, our system automatically selects a guideline-based, standardized followup schema using the TNM-Classification. We found only one app, the "survivor care app" [14], which can read a QR code in which a personalized care plan is encoded. However, this care plan needs to be created by the physician and the app has not been updated since 3 years.

With its current functionalities, our app is only used in phases when the patient has active appointments which is a limitation. To ensure a continuous use, additional functionalities should be integrated. The storage of vital parameters already addresses this issue. In future work, an automatic data upload from medical devices or gadgets should be implemented to simplify the usage of this feature and to ensure the long-time use of the app as a follow-up care tool. In addition to the information page, additional components for empowerment could be integrated such as patient-to-patient services [2] or patient portal services [13]. For example, the patient could be enabled to record the health status on a weekly or even daily basis and the app could integrate values from smart devices. This would allow a continuous health monitoring, not only for the patient, but also for the physician and could contribute to the self-management capabilities of a patient. Self-management is an essential but frequently neglected component of

\begin{tabular}{|c|c|c|c|c|c|}
\hline & Does not apply & Does partially apply & Neither/nor & Applies mostly & Applies completely \\
\hline It is easy for me to learn how to use the app & 0 & 0 & 2 & 4 & 3 \\
\hline I find it easy to use the app & 0 & 0 & 2 & 4 & 3 \\
\hline I perceive the app as easy to use & 0 & 0 & 3 & 4 & 2 \\
\hline The terms used are clear to me & 0 & 0 & 0 & 6 & 3 \\
\hline The use of symbols are clear to me & 0 & 0 & 1 & 3 & 5 \\
\hline I perceive the app as clear and understandable & 0 & 0 & 3 & 4 & 2 \\
\hline The use of the app does not require too much mental effort & 0 & 0 & 1 & 5 & 3 \\
\hline $\begin{array}{l}\text { When I use the app the first time menus and functionalities } \\
\text { are clear at the first instant }\end{array}$ & 0 & 0 & 1 & 4 & 4 \\
\hline I can find all the relevant information & 0 & 0 & 2 & 1 & 6 \\
\hline I like the design of the app & 0 & 0 & 0 & 7 & 2 \\
\hline
\end{tabular}

Table 3: Multiple choice questionnaire for the usability test. The total number of selections per scale item is shown received from all nine persons. 
chronic illness management [16]. However, O’Neill and Brady found out by reviewing 68 colorectal-themed smartphone apps that most of them are intended for educational purposes, and only few of them offer symptom diaries or other self-management functionalities.

A potential handling problem with our app could be that the patient enters the data on the tumor incorrectly resulting in a wrong schema that is loaded. The correct TNM input is central to a successful use. Therefore, we designed a printed data sheet to assist the patient in entering his data. However, the physician must agree to fill this sheet and explain the data briefly to the patient. Otherwise, the app may be misapplied. In future, it could be envisioned to create a barcoded information sheet that could be scanned by the app to prevent faulty entries. A clear benefit of our approach is that physicians were involved right from the beginning which increases the recommendation of the app to patients. This was found by O'Neill and Brady. as an important issue, since most available apps have been developed without involvement of health professionals [16].

\section{Limitations of the study design}

From the usability test results, we conclude that the app provides a good basis for a successful product after some extensions. The test persons liked the look and feel of the user interface, the design in general and the ease of use. Suggestions for improvements have been collected from users and concern the presentation of the appointment schedule and some GUI elements.

The usability test was performed with subjects from the author's social environment. Therefore, the results might be biased. Feedback was only collected from one patient and one physician which is a very limited study design. The objective was to collect input for improving the app and running a pre-test of the usability study to collect input for improvements on the app and study design. The usability study with test scenario and feedback provided valuable suggestions for improving the mobile application. After realizing the improvements, the usability test will be repeated with additional test subjects. Also a clinical study will be planned to study the long-term effects. This will help to identify potential barriers in using the app.

We only assessed the usability. It would be interesting, to assess the effects of using IT to improve follow-up cancer care. At least, Groen et al. [13] found out that IT can be useful in this process. Additional comprehensive studies are still required to verify this.

\section{Conclusion}

We presented a mobile system that provides a care plan and relevant information to cancer survivors. In future work, the app will be extended by additional features such as export of vital parameters and timeline as PDF and integration of vital parameters directly via Bluetooth or directly by integrating the corresponding web service from MIDATA. The platform already provides services the enabling an upload of data from Withings and Fitbit. The data could be collected from MIDATA and shown in the tumor care app. Additional features to be implemented are entering the family history, anamnesis, and storing of documents such as discharge summaries or laboratory results. Another extension includes adding a schema for chemo therapy and radiation therapy for creating additional reminders.

\section{References}

1. Philipps JL, Currow DC (2010) Cancer as a chronic disease. Collegian 17 47-50.

2. https://www.bag.admin.ch/bag/de/home/service/zahlen-fakten/zahlen-faktenzu-spitaelern/qualitaetsindikatoren-der-schweizer-akutspitaeler.html

3. http://www.sggssg.ch/fileadmin/_migrated/content_uploads/CRC_SGG_ September_2014_DE_01.pdf

4. Viehl CT (2010) Inadequate quality of surveillance after curative surgery for colon cancer. Ann Surg Oncol 17: 2663-2669.

5. Ventola CL (2014) Mobile devices and apps for health care professionals: use and benefits. P\&T 39: 356-364

6. Price M, Yuen EK, Goetter EM, Herbert JD, Forman EM, et al. (2014) mHealth a mechanism to deliver more accessible, more effective mental health care. Clin Psychol Psychother 21: 427-436.

7. http://www.nachsorge-app.de/

8. https://itunes.apple.com/us/app/my-care-plan-cancer-survivors/ id821784862?mt=8 last access: 11.03 .2017

9. https://www.krebshilfe-wien.at/services/krebshilfe-app/

10. Buzzacchino S, Lanzola G, Bossi P, Licitra L, Quaglini S (2015) A mobile application supporting outpatient treatment and follow-up. Stud Health Technol Inform 210: 788-792.

11. Rubin J, Chisnell JD (2008) Handbook of usability testing: how to plan, design and conduct effective tests. Aufl: John Wiley \& Sons.

12. Hewitt M, Greenfield S, Stovall E (2006) From cancer patient to cancer survivor: Lost in translation. National Academy Press, Washington, DC, USA.

13. Groen WG, Kuijpers W, Oldenburg HS, Wouters MW, Aaronson NK, et al (2015) Empowerment of cancer survivors through information technology: an integrative review. J Med Internet Res 17: e270.

14. Tsamaloukas A (2014) Correspondence (letter to the editor): App for Aftercare. Dtsch Arztebl Int 111: 403

15. Geng Y, Sahit M (2015) "Patient engagement in cancer survivorship care through mHealth: A consumer-centered review of existing mobile applications." AMIA Annual Symposium Proceedings 2015: 580-588.

16. O'Neill S, Brady RR (2012) Colorectal smartphone apps: opportunities and risks. Colorectal Dis 14: 530-534.

17. Pandey A, Hasan S, Dubey D, Sarangi S (2013) Smartphone apps as a source of cancer information: changing trends in health information-seeking behavior. J Cancer Educ 28: 138-142. 